\title{
A model for the propagation and scattering of ultrasound in tissue
}

Jensen, Jørgen Arendt

Published in:

Acoustical Society of America. Journal

Link to article, DOI:

$10.1121 / 1.400497$

Publication date:

1991

Document Version

Publisher's PDF, also known as Version of record

Link back to DTU Orbit

Citation (APA):

Jensen, J. A. (1991). A model for the propagation and scattering of ultrasound in tissue. Acoustical Society of America. Journal, 89(1), 182-190. https://doi.org/10.1121/1.400497

\section{General rights}

Copyright and moral rights for the publications made accessible in the public portal are retained by the authors and/or other copyright owners and it is a condition of accessing publications that users recognise and abide by the legal requirements associated with these rights.

- Users may download and print one copy of any publication from the public portal for the purpose of private study or research.

- You may not further distribute the material or use it for any profit-making activity or commercial gain

- You may freely distribute the URL identifying the publication in the public portal

If you believe that this document breaches copyright please contact us providing details, and we will remove access to the work immediately and investigate your claim 


\title{
A model for the propagation and scattering of ultrasound in tissue
}

\author{
Jørgen Arendt Jensen \\ Electronics Institute, Building 349, Technical University of Denmark, 2800 Lyngby, Denmark
}

\author{
(Received 5 April 1990; accepted for publication 3 August 1990)
}

\begin{abstract}
An inhomogeneous wave equation is derived describing propagation and scattering of ultrasound in an inhomogeneous medium. The scattering term is a function of density and propagation velocity perturbations. The integral solution to the wave equation is combined with a general description of the field from typical transducers used in clinical ultrasound to yield a model for the received pulse-echo pressure field. Analytic expressions are found in the literature for a number of transducers, and any transducer excitation can be incorporated into the model. An example is given for a concave, nonapodized transducer in which the predicted pressure field is compared to a measured field.
\end{abstract}

PACS numbers: 43.35.Bf, 43.80.Qf

\section{INTRODUCTION}

Ultrasound is used with great success in the diagnosis of abnormalities in soft tissue structures in the human body. Cross-sectional pictures are made in real time with the $B$ mode scan technique by current scanners. A high picture quality is obtained by employing linear and phased array transducers, and by post-processing the envelope detected signal from the transducer. But the ultrasound pictures still lack contrast and resolution compared to $x$-ray and NMR (nuclear magnetic resonance) pictures. It does not seem possible to cure this deficiency with the current techniques based on analog electronics, and it therefore seems plausible that future scanners will use high-frequency sampling of the transducer signal in order to employ digital signal processing.

Developing algorithms that take advantage of this sampling necessitates quantitative knowledge of the received pulse-echo pressure field. The object of this paper is to develop such a model for the received pressure field.

In medical ultrasound, a pulse is emitted into the body and is scattered and reflected by density and propagation velocity perturbations. The received field can be found by solving an appropriate wave equation. This has been done in a number of papers. ${ }^{1,2}$ Gore and Leeman ${ }^{1}$ considered a wave equation where the scattering term was a function of the adiabatic compressibility and the density. The transducer was modeled by an axial and lateral pulse that were separable. Fatemi and $\mathrm{Kak}^{2}$ used a wave equation where the scattering only originated from velocity fluctuations, and the transducer was restricted to be circularly symmetric and unfocused (flat).

The scattering term for the wave equation used in this paper is a function of density and propagation velocity perturbations, and the wave equation is equivalent to the one used by Gore and Leeman. ${ }^{1}$ No restrictions are enforced on the transducer geometry or its excitation, and analytic expressions for a number of geometries can be incorporated into the model.

The model includes attenuation due to propagation and scattering, but not the dispersive attenuation observed for propagation in tissue. This can, however, be incorporated into the model as indicated in Sec. VI.

The paper is organized as follows. The following section derives the wave equation and describes the different linearity assumptions made. Section II calculates the scattered field and Sec. III introduces the transducer model for calculation of the incident field. Section IV combines the wave equation solution and the transducer model to give the final equation for the received pressure field. To indicate the precision of the model, examples of predicted pressure fields compared to measured fields are given in Sec. V. The paper is concluded in Sec. VI.

\section{DERIVATION OF THE WAVE EQUATION}

In this section, we derive the wave equation. The section has been included in order to explain, in detail, the different linearity assumptions and approximations made. To obtain a solvable wave equation, some assumptions and approximations must be made. The first one states that the instantaneous acoustic pressure and density can be written as

$$
\begin{aligned}
& P_{\text {ins }}(\mathbf{r}, t)=P+p_{1}(\mathbf{r}, t), \\
& \rho_{\text {ins }}(\mathbf{r}, t)=\rho(\mathbf{r})+\rho_{1}(\mathbf{r}, t),
\end{aligned}
$$

in which $P$ is the mean pressure of the medium and $\rho$ is the density of the undisturbed medium. The pressure variation $p_{1}$ is caused by the ultrasound wave and is considered small compared to $P$. The density change caused by the wave is $\rho_{1}$. Both $p_{1}$ and $\rho_{1}$ are small quantities of first order.

Our second assumption is that no heat conduction or conversion of ultrasound to thermal energy take place. Thus, the entropy is constant for the process, so the acoustic pressure and density satisfy the adiabatic equation: ${ }^{3}$

$$
\frac{d P_{\mathrm{ins}}}{d t}=c^{2} \frac{d \rho_{\mathrm{ins}}}{d t}
$$

The equation contains total derivatives, as the relation is satisfied for a given particle of the tissue rather than at a given point in space. This is the Lagrange description of the motion. ${ }^{4}$ For our purpose, the Euler description is more appropriate. Here, the coordinate system is fixed in space and 
the equation describes the properties of whatever particle of fluid there is at a given point at a given time. Converting to a Euler description results in the following constitutive equation: ${ }^{3,4}$

$$
\frac{1}{c^{2}} \frac{\partial p_{1}}{\partial t}=\frac{\partial \rho_{1}}{\partial t}+\mathbf{u} \cdot \nabla \rho
$$

using the fact that $P$ and $\rho$ do not depend on time and that $\rho_{1}$ is small compared to $\rho . u$ is the particle velocity, $\nabla$ is the gradient operator, and $\cdot$ symbolizes the scalar product.

The pressure, density, and particle velocity must also satisfy the hydrodynamical equations: ${ }^{3}$

$$
\begin{aligned}
& \rho_{\text {ins }} \frac{d \mathbf{u}}{d t}=-\nabla P_{\mathrm{ins}}, \\
& \frac{\partial \rho_{\mathrm{ins}}}{\partial t}=-\nabla \cdot\left(\rho_{\mathrm{ins}} \mathbf{u}\right),
\end{aligned}
$$

which are the dynamic equation and the equation of continuity. Using (1) and (2) and discarding higher-order terms we can write

$$
\begin{aligned}
& \rho \frac{\partial \mathbf{u}}{\partial t}=-\nabla p_{1}, \\
& \frac{\partial \rho_{1}}{\partial \mathrm{t}}=-\nabla \cdot(\rho \mathbf{u}) .
\end{aligned}
$$

Differentiating (8) with respect to $t$ and inserting (7) gives

$$
\frac{\partial^{2} \rho_{1}}{\partial^{2} t}=-\nabla \cdot\left(\rho \frac{\partial \mathbf{u}}{\partial t}\right)=-\nabla \cdot\left(-\nabla p_{1}\right)=\nabla^{2} p_{1} .
$$

Differentiating (4) with respect to $t$ gives

$$
\frac{1}{c^{2}} \frac{\partial^{2} p_{1}}{\partial^{2} t}=\frac{\partial^{2} \rho_{1}}{\partial^{2} t}+\frac{\partial \mathbf{u}}{\partial t} \cdot \nabla \rho
$$

and inserting (9) and (7) leads to

$$
\nabla^{2} p_{1}-\frac{1}{c^{2}} \frac{\partial^{2} p_{1}}{\partial^{2} t}=\frac{1}{\rho} \nabla \rho \cdot \nabla p_{1} .
$$

We now assume that the propagation velocity and the density only vary slightly from their mean values, so that

$$
\begin{aligned}
& \rho(\mathbf{r})=\rho_{0}+\Delta \rho(\mathbf{r}), \\
& c(\mathbf{r})=c_{0}+\Delta c(\mathbf{r}),
\end{aligned}
$$

where $\rho_{0} \gg \Delta \rho$ and $c_{0} \gg \Delta c$. Then,

$$
\begin{aligned}
\nabla^{2} p_{1} & -\frac{1}{\left(c_{0}+\Delta c\right)^{2}} \frac{\partial^{2} p_{1}}{\partial^{2} t} \\
& =\frac{1}{\left(\rho_{0}+\Delta \rho\right)} \nabla\left(\rho_{0}+\Delta \rho\right) \cdot \nabla p_{1} .
\end{aligned}
$$

Ignoring small quantities of second order and using the approximation

$$
1 /(1+\Delta) \approx 1-\Delta
$$

we get

$$
\begin{aligned}
\nabla^{2} p_{1} & -\left(\frac{1}{c_{0}^{2}}-\frac{2 \Delta c}{c_{0}^{3}}\right) \frac{\partial^{2} p_{1}}{\partial^{2} t} \\
= & \left(\frac{1}{\rho_{0}} \nabla(\Delta \rho)-\frac{\Delta \rho}{\rho_{0}^{2}} \nabla(\Delta \rho)\right) \cdot \nabla p_{1} .
\end{aligned}
$$

Neglecting the second-order term $\left(\Delta \rho / \rho_{0}^{2}\right) \nabla(\Delta \rho) \cdot \nabla p_{1}$, we finally get the wave equation:

$$
\nabla^{2} p_{1}-\frac{1}{c_{0}^{2}} \frac{\partial^{2} p_{1}}{\partial t^{2}}=-\frac{2 \Delta c}{c_{0}^{3}} \frac{\partial^{2} p_{1}}{\partial t^{2}}+\frac{1}{\rho_{0}} \nabla(\Delta \rho) \cdot \nabla p_{1} .
$$

The two terms on the right side of the equation are the scattering terms that vanish for a homogeneous medium. The wave equation was derived in Chernov. ${ }^{3}$ It has also been considered in Gore and Leeman ' and Morse and Ingard ${ }^{4}$ in a slightly different form, where the scattering terms were a function of the adiabatic compressibility $\kappa$ and the density.

\section{CALCULATION OF THE SCATTERED FIELD}

Having derived a suitable wave equation, we now calculate the scattered field from a small inhomogeneity embedded in a homogeneous surrounding. The scene is depicted in Fig. 1.

The inhomogeneity is identified by $\mathbf{r}_{1}$ and enclosed in the volume $V^{\prime}$. The scattered field is calculated at the point indicated by $\mathbf{r}_{2}$ by integrating all the spherical waves emanating from the scattering region $V^{\prime}$ using the time-dependent Green's function for unbounded space. Thus, the scattered field is: ${ }^{1,4}$

$$
\begin{aligned}
p_{s}\left(\mathbf{r}_{2}, t\right)= & \int_{V} \int_{T}\left[\frac{1}{\rho_{0}} \nabla\left[\Delta \rho\left(\mathbf{r}_{1}\right)\right] \cdot \nabla p_{1}\left(\mathbf{r}_{1}, t_{1}\right)\right. \\
& \left.-\frac{2 \Delta c\left(\mathbf{r}_{1}\right)}{c_{0}^{3}} \frac{\partial^{2} p_{1}\left(\mathbf{r}_{1}, t_{1}\right)}{\partial t^{2}}\right] \\
& \times G\left(\mathbf{r}_{1}, t_{1} \mid \mathbf{r}_{2}, t\right) d t_{1} d^{3} \mathbf{r}_{1}
\end{aligned}
$$

where $G$ is Green's function:

$$
G\left(\mathbf{r}_{1}, t_{1} \mid \mathbf{r}_{2}, t\right)=\frac{\delta\left(t-t_{1}-\left|\mathbf{r}_{2}-\mathbf{r}_{1}\right| / c_{0}\right)}{4 \pi\left|\mathbf{r}_{2}-\mathbf{r}_{1}\right|},
$$

$d^{3} \mathbf{r}_{1}$ means integrating with respect to $\mathbf{r}_{1}$ over the volume $V^{\prime}$, and $T$ denotes integration over time.

We denote by

Homogeneous medium

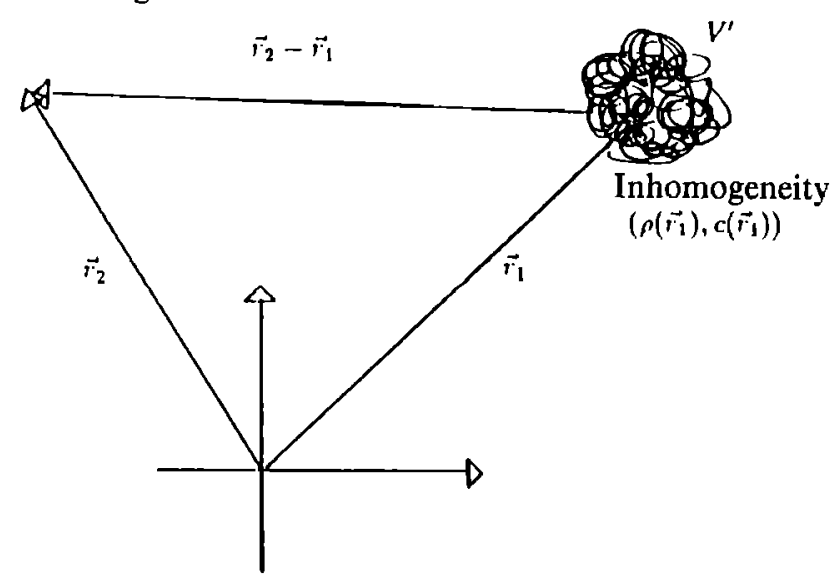

FIG. 1. Coordinate system for calculating the scattered field. 


$$
F_{\mathrm{op}}=\frac{1}{\rho_{0}} \nabla\left[\Delta \rho\left(\mathbf{r}_{1}\right)\right] \cdot \nabla-\frac{2 \Delta c\left(\mathbf{r}_{1}\right)}{c_{0}^{3}} \frac{\partial^{2}}{\partial t^{2}},
$$

the scattering operator.

The pressure field inside the scattering region is

$$
p_{1}(\mathbf{r}, t)=p_{i}(\mathbf{r}, t)+p_{s}(\mathbf{r}, t),
$$

where $p_{i}$ is the incident pressure field. As can be seen, the integral cannot be solved directly. To solve it, we apply the Born-Neumann expansion. ${ }^{5}$ If $G_{i}$ symbolizes the integral operator representing Green's function and the integration, and $F_{\text {op }}$ the scattering operator, then the first-order Born approximation can be written as

$$
p_{s_{1}}\left(\mathbf{r}_{2}, t\right)=G_{i} F_{\text {op }} p_{i}\left(\mathbf{r}_{1}, t_{1}\right) \text {. }
$$

Here, $p_{s}$ has been set to zero in (20). Inserting $p_{s_{1}}$ in (20), and then in (17), we arrive at

$$
\begin{aligned}
p_{s_{2}} & \left(\mathbf{r}_{2}, t\right) \\
& =G_{i} F_{\mathrm{op}}\left[p_{i}\left(\mathbf{r}_{1}, t_{1}\right)+G_{i} F_{\mathrm{op}} p_{i}\left(\mathbf{r}_{1}, t_{1}\right)\right] \\
& =G_{i} F_{\mathrm{op}} p_{i}\left(\mathbf{r}_{1}, t_{1}\right)+\left(G_{i} F_{\mathrm{op}}\right)^{2} p_{i}\left(\mathbf{r}_{1}, t_{1}\right) .
\end{aligned}
$$

It is emphasized here that $G_{i}$ indicates an integral over $\mathbf{r}_{1}$ and $t_{1}$, and not the pressure at point $\mathbf{r}_{1}$ and time $t_{1}$ but over the volume of $V^{\prime}$ and time $T$ indicated by $\mathbf{r}_{1}$ and $t_{1}$.

The general expression for the scattered field, then, is:

$$
\begin{aligned}
p_{s}\left(\mathbf{r}_{2}, t\right)= & G_{i} F_{\mathrm{op}} p_{i}\left(\mathbf{r}_{1}, t_{1}\right) \\
& +\left(G_{i} F_{\mathrm{op}}\right)^{2} p_{i}\left(\mathbf{r}_{1}, t_{1}\right) \\
& +\left(G_{i} F_{\mathrm{op}}\right)^{3} p_{i}\left(\mathbf{r}_{1}, t_{1}\right) \\
& +\left(G_{i} F_{\mathrm{op}}\right)^{4} p_{i}\left(\mathbf{r}_{1}, t_{1}\right)+\cdots .
\end{aligned}
$$

Terms involving $\left(G_{i} F_{\mathrm{op}}\right)^{N} p_{i}\left(\mathbf{r}_{1}, t_{1}\right)$, where $N>1$, describe multiple scattering of order $N$. Usually the scattering from small obstacles is considered weak, so higher-order terms can be neglected. Thus, a useful approximation is to employ only the first term in the expansion. This corresponds to the first-order Born approximation.

Using this, (17) can be approximated by

$$
\begin{aligned}
p_{s}\left(\mathbf{r}_{2}, t\right) \approx & \int_{V^{\prime}} \int_{T}\left(\frac{1}{\rho_{0}} \nabla\left[\Delta \rho\left(\mathbf{r}_{1}\right)\right] \cdot \nabla p_{i}\left(\mathbf{r}_{1}, t_{1}\right)\right. \\
& \left.-\frac{2 \Delta c\left(\mathbf{r}_{1}\right)}{c_{0}^{3}} \frac{\partial^{2} p_{i}\left(\mathbf{r}_{1}, t_{1}\right)}{\partial t^{2}}\right) \\
& \times G\left(\mathbf{r}_{1}, t_{1} \mid \mathbf{r}_{2}, t\right) d t_{1} d^{3} \mathbf{r}_{1} .
\end{aligned}
$$

So, in order to calculate the scattered field, the incident field for the homogeneous medium must be calculated.

\section{CALCULATION OF THE INCIDENT FIELD}

The incident field is generated by the ultrasound transducer, assuming no other sources exist in the tissue. We want to calculate the field of a typical focused ultrasound transducer generating a transient field.

A review of different methods to calculate this can be found in Harris. ${ }^{6,7}$ The most elegant, accurate, and straightforward solution is to use the convolution method developed by Tupholme ${ }^{8}$ and Stepanishen. ${ }^{9,10}$

By this method the incident field is found by solving the wave equation for the homogeneous case:

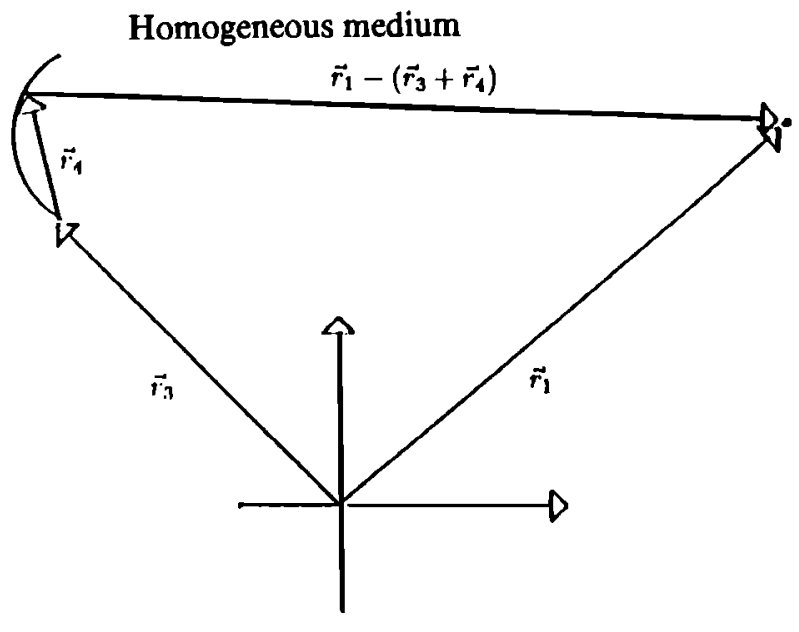

FIG. 2. Coordinate system for calculating the incident field.

$$
\nabla^{2} p_{1}-\frac{1}{c_{0}^{2}} \frac{\partial^{2} p_{1}}{\partial^{2} t}=0
$$

The field is conveniently calculated by employing the velocity potential $\psi(r, t)$, and enforcing appropriate boundary conditions. ${ }^{8,9}$ The velocity potential satisfies the following wave equation for the homogeneous medium:

$$
\nabla^{2} \psi-\frac{1}{c_{0}^{2}} \frac{\partial^{2} \psi}{\partial^{2} t}=0
$$

and the pressure is calculated from

$$
p(\mathbf{r}, t)=\rho_{0} \frac{\partial \psi(\mathbf{r}, t)}{\partial t} .
$$

The coordinate system shown in Fig. 2 is used in the calculation. The particle velocity normal to the transducer surface is denoted by $v\left(\mathbf{r}_{3}+\mathbf{r}_{4}, t\right)$.

The solution to the homogeneous wave equation is ${ }^{9}$

$$
\begin{aligned}
\psi\left(\mathbf{r}_{1}\right. & \left.+\mathbf{r}_{3}, t\right) \\
& =\int_{S} \int_{T} v\left(\mathbf{r}_{3}+\mathbf{r}_{4}, t_{3}\right) g\left(\mathbf{r}_{1}, t \mid \mathbf{r}_{3}+\mathbf{r}_{4}, t_{3}\right) d t_{3} d^{2} \mathbf{r}_{4}
\end{aligned}
$$

when the transducer is mounted in a rigid infinite planar baffle. Here, $S$ denotes the transducer surface.

The Green's function for a bounded medium is $g$ and is

$$
\begin{aligned}
& g\left(\mathbf{r}_{1}, t \mid \mathbf{r}_{3}+\mathbf{r}_{4}, t_{3}\right) \\
& \quad=\delta\left(t-t_{3}-\left|\mathbf{r}_{1}-\mathbf{r}_{3}-\mathbf{r}_{4}\right| / c_{0}\right) /\left(2 \pi\left|\mathbf{r}_{1}-\mathbf{r}_{3}-\mathbf{r}_{4}\right|\right) .
\end{aligned}
$$

The distance from $S$ to the point where the field is calculated is $\left|\mathbf{r}_{1}-\mathbf{r}_{3}-\mathbf{r}_{4}\right|$ and $c_{0}$ is the mean propagation velocity. The field is calculated under the assumption of radiation into an isotropic, homogeneous, nondissipative medium. The integral is a statement of Huygens' principle that, for a planar vibrating surface, each point on the source generates a 
spherical wave, and the resulting field is found by integrating these waves at the point of interest.

If a slightly curved transducer is used, an additional term is introduced, as shown in Morse and Feshbach. " This term is called the second-order diffraction term in Penttinen and Luukkala. ${ }^{12}$ It can be shown to vanish for a planar transducer, and as long as the transducer is only slightly curved and large compared to the wavelength of the ultrasound, the resulting expression is a good approximation to the pressure field. ${ }^{12}$

If we assume the particle velocity to be uniform over the surface of the transducer, $(28)$ can be reduced to ${ }^{10}$

$$
\begin{aligned}
& \psi\left(\mathbf{r}_{1}, \mathbf{r}_{3}, t\right) \\
& \quad=\int_{T} v\left(t_{3}\right) \int_{S} g\left(\mathbf{r}_{1}, t \mid \mathbf{r}_{3}+\mathbf{r}_{4}, t_{3}\right) d^{2} \mathbf{r}_{4} d t_{3} .
\end{aligned}
$$

The function

$$
\begin{aligned}
h\left(\mathbf{r}_{1}, \mathbf{r}_{3}, t-t_{3}\right) & =\int_{S} g\left(\mathbf{r}_{1}, t \mid \mathbf{r}_{3}+\mathbf{r}_{4}, t_{3}\right) d^{2} \mathbf{r}_{4} \\
& =\int_{S} \frac{\delta\left(t-t_{3}-\left|\mathbf{r}_{1}-\mathbf{r}_{3}-\mathbf{r}_{4}\right| / c_{0}\right)}{2 \pi\left|\mathbf{r}_{1}-\mathbf{r}_{3}-\mathbf{r}_{4}\right|} d^{2} \mathbf{r}_{4}
\end{aligned}
$$

is called the spatial impulse response and it relates the transducer geometry to the acoustical field. By this function, we can write

$$
\psi\left(\mathbf{r}_{1}, \mathbf{r}_{3}, t\right)=v(t) \star h\left(\mathbf{r}_{1}, \mathbf{r}_{3}, t\right) .
$$

The piston velocity waveform is $v(t)$, and the velocity potential is written as a convolution in time between this and the spatial impulse response. Note that $h$ depends on the difference between $r_{1}$ and $\mathbf{r}_{3}$, thus it is spatially varying. To emphasize this, $h$ is written $h\left(\mathbf{r}_{1}, \mathbf{r}_{3}, t\right)$.

The sound pressure for the incident field, then, is

$$
\begin{aligned}
p\left(\mathbf{r}_{1}, \mathbf{r}_{3}, t\right) & =\rho_{0} \frac{\partial \psi\left(\mathbf{r}_{1}, \mathbf{r}_{3}, t\right)}{\partial t} \\
& =\rho_{0} v(t) \star \frac{\partial h\left(\mathbf{r}_{1}, \mathbf{r}_{3}, t\right)}{\partial t}
\end{aligned}
$$

or

$$
p\left(\mathbf{r}_{1}, \mathbf{r}_{3}, t\right)=\rho_{0} \frac{\partial v(t)}{\partial t} \star h\left(\mathbf{r}_{1}, \mathbf{r}_{3}, t\right) .
$$

Note, the separation between the excitation and the transducer geometry. Here, $v(t)$ includes the electromechanical impulse response of the transducer. ${ }^{13,14}$

Explicit solutions for a number of transducer geometries have been found. Analytical expressions for a circular, flat transducer can be found in Stepanishen, ${ }^{9}$ and for a circular, concave geometry in Penttinen and Luukkala. ${ }^{12}$

It must be emphasized that the only approximation introduced here is the assumption of separability between the excitation and the transducer geometry. Transducers can be constructed in which this is a very good approximation, so that the pressure field calculated by this method is in good agreement with the measured field.

\section{CALCULATION OF THE RECEIVED SIGNAL}

The received signal is the scattered pressure field integrated over the transducer surface, convolved with the electromechanical impulse response $E_{m}(t)$ of the transducer. To calculate this, we introduce the coordinate system shown in Fig. 3.

The term $r_{6}+r_{5}$ indicates a receiving element on the surface of the transducer that is located at $\mathbf{r}_{5}$. The received signal is

$$
p_{r}\left(\mathbf{r}_{5}, t\right)=E_{m}(t) \star \int_{s} p_{s}\left(\mathbf{r}_{6}+\mathbf{r}_{5}, t\right) d^{2} \mathbf{r}_{6} .
$$

The scattered field is

$$
\begin{aligned}
& p_{s}\left(\mathbf{r}_{6}+\mathbf{r}_{5}, t\right) \\
&=\frac{1}{2} \int_{V^{\prime}} \int_{T} F_{\mathrm{op}}\left[p_{i}\left(\mathbf{r}_{1}, t_{1}\right)\right] \\
& \times \frac{\delta\left(t-t_{1}-\left|\mathbf{r}_{6}+\mathbf{r}_{5}-\mathbf{r}_{1}\right| / c_{0}\right)}{2 \pi\left|\mathbf{r}_{6}+\mathbf{r}_{5}-\mathbf{r}_{1}\right|} d t_{1} d^{3} \mathbf{r}_{1} .
\end{aligned}
$$

Combining (36) with (35) and comparing with (31), we see that $p$, includes Green's function for bounded space integrated over the transducer surface, which is equal to the spatial impulse response. Inserting the expression for $p_{i}$ and performing the integration over the transducer surface and over time, results in

$$
\begin{aligned}
p_{r}\left(\mathbf{r}_{5}, t\right)= & E_{m}(t) \star \frac{1}{2} \int_{V \cdot} F_{\mathrm{op}}\left(\rho_{0} \frac{\partial v(t)}{\partial t} \star h\left(\mathbf{r}_{1}, \mathbf{r}_{3}, t\right)\right) \\
& \times \star h\left(\mathbf{r}_{5}, \mathbf{r}_{1}, t\right) d^{3} \mathbf{r}_{1} .
\end{aligned}
$$

If the position of the transmitting and the receiving transducer is the same $\left(r_{3}=r_{5}\right)$, then a simple rearrangement of (37) yields

$$
\begin{aligned}
p_{r}\left(\mathbf{r}_{5}, t\right)= & \frac{\rho_{0}}{2} E_{m}(t) \star \frac{\partial v(t)}{\partial t} \\
& \times \int_{V^{\cdot}} F_{\mathrm{op}}\left[H_{\mathrm{pe}}\left(\mathbf{r}_{1}, \mathbf{r}_{5}, t\right)\right] d^{3} \mathbf{r}_{1},
\end{aligned}
$$

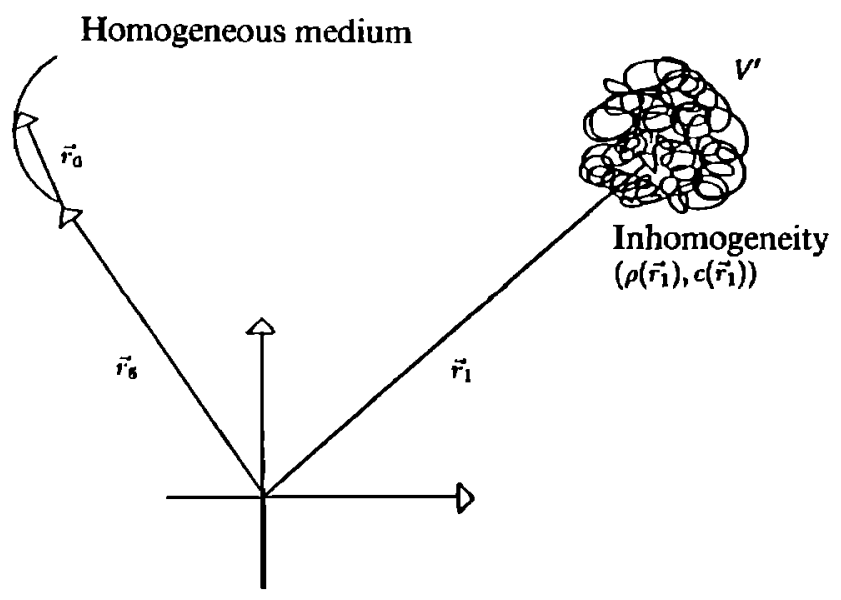

FIG. 3. Coordinate system for calculating the received signal. 
where

$$
H_{\mathrm{pe}}\left(\mathbf{r}_{1}, \mathbf{r}_{5}, t\right)=h\left(\mathbf{r}_{1}, \mathbf{r}_{5}, t\right) \star h\left(\mathbf{r}_{5}, \mathbf{r}_{1}, t\right)
$$

is the pulse-echo spatial impulse response.

The calculated signal is the response measured for one given position of the transducer. For a $B$-mode scan picture, a number of scan-lines are measured and combined to a picture. To analyze this situation, the last factor in (38) is explicitly written out as

$$
\begin{aligned}
\int_{V^{\prime}} & \left(\frac{1}{\rho_{0}} \nabla\left[\Delta \rho\left(\mathbf{r}_{1}\right)\right] \cdot \nabla H_{\mathbf{p e}}\left(\mathbf{r}_{1}, \mathbf{r}_{5}, t\right)\right. \\
& \left.-\frac{2 \Delta c\left(\mathbf{r}_{1}\right)}{c_{0}^{3}} \frac{\partial^{2} H_{\mathrm{pe}}\left(\mathbf{r}_{1}, \mathbf{r}_{5}, t\right)}{\partial t^{2}}\right) d^{3} \mathbf{r}_{1} .
\end{aligned}
$$

From Sec. III, we know that $H_{\mathrm{pe}}$ is a function of the distance between $r_{1}$ and $r_{5}$, while $\Delta \rho$ and $\Delta c$ only are functions of $r_{1}$. So, when $r_{5}$ is varied over the volume of interest, the resulting image is a spatial nonstationary convolution between $\Delta \rho$, $\Delta c$, and a modified form of the pulse-echo spatial impulse response.

If we assume that the pulse-echo spatial impulse is slowly varying so that the spatial frequency content is constant over a finite volume, then (40) can be rewritten as

$$
\begin{aligned}
\int_{V} & {\left[\frac{1}{\rho_{0}} \Delta \rho\left(\mathbf{r}_{1}\right) \nabla^{2} H_{\mathrm{pe}}\left(\mathbf{r}_{1}, \mathbf{r}_{5}, t\right)\right.} \\
& \left.-\frac{2 \Delta c\left(\mathbf{r}_{1}\right)}{c_{0}^{3}} \frac{\partial^{2} H_{\mathrm{pe}}\left(\mathbf{r}_{1}, \mathbf{r}_{5}, t\right)}{\partial t^{2}}\right] d^{3} \mathbf{r}_{1} .
\end{aligned}
$$

Here, $H_{\mathrm{pc}}$ is a function of the distance between the transducer and the scatterer, or equivalently, of the corresponding time given by

$$
t=\left|\mathbf{r}_{1}-\mathbf{r}_{s}\right| / c_{0} .
$$

The Laplace operator is the second derivative with respect to the distance, which in this case corresponds to the second derivative with respect to time. So,

$$
\nabla^{2} H_{\mathrm{pe}}\left(\mathrm{r}_{1}, \mathrm{r}_{5}, t\right)=\frac{1}{c_{0}^{2}} \frac{\partial^{2} H_{\mathrm{pe}}\left(\mathrm{r}_{1}, \mathrm{r}_{5}, t\right)}{\partial t^{2}}
$$

assuming only small deviations from the mean propagation velocity.

Using these approximations, (38) can be rewritten as $p_{r}\left(\mathbf{r}_{5}, t\right)$

$$
\begin{aligned}
= & \frac{\rho_{0}}{2} E_{m}(t) * \frac{\partial v(t)}{\partial t} * \int_{V^{\prime}}\left(\frac{\Delta \rho\left(\mathbf{r}_{1}\right)}{\rho_{0}}-\frac{2 \Delta c\left(\mathbf{r}_{1}\right)}{c_{0}}\right) \\
& \times \frac{1}{c_{0}^{2}} \frac{\partial^{2} H_{\mathrm{pe}}\left(\mathbf{r}_{1}, \mathbf{r}_{5}, t\right)}{\partial t^{2}} d^{3} \mathbf{r}_{1} .
\end{aligned}
$$

Symbolically, this is written as

$$
p_{r}\left(\mathbf{r}_{5}, t\right)=v_{\mathrm{pe}}(t) \star f_{t}\left(\mathbf{r}_{1}\right) \star h_{\mathrm{pe}}\left(\mathbf{r}_{1}, \mathbf{r}_{5}, t\right) .
$$

Here, * denotes spatial convolution. The pulse-echo wavelet is $v_{\text {pe }}$, which includes the transducer excitation and the electromechanical impulse response during emission and reception of the pulse. The term $f_{m}$ accounts for the inhomogeneities in the tissue due to density and propagation velocity perturbations that give rise to the scattered signal. The term $h_{\text {pe }}$ is the modified pulse-echo spatial impulse response that relates the transducer geometry to the spatial extent of the scattered field. Explicitly written out, these terms are

$$
\begin{aligned}
& v_{\mathrm{pe}}(t)=\frac{\rho_{0}}{2} E_{m}(t) \star \frac{\partial v(t)}{\partial t}, \\
& f_{m}\left(\mathbf{r}_{1}\right)=\Delta \rho\left(\mathbf{r}_{1}\right) / \rho_{0}-2 \Delta c\left(\mathbf{r}_{1}\right) / c_{0}, \\
& h_{\mathrm{pe}}\left(\mathbf{r}_{1}, \mathbf{r}_{5}, t\right)=\frac{1}{c_{0}^{2}} \frac{\partial^{2} H_{\mathrm{pe}}\left(\mathbf{r}_{1}, \mathbf{r}_{5}, t\right)}{\partial t^{2}} .
\end{aligned}
$$

Expression (45) consists of three distinct terms. The interesting signal, and the one that should be displayed in medical ultrasound, is $f_{m}\left(r_{1}\right)$. We, however, measure a time and spatially smoothed version of this, which obscures the finer details in the picture. The smoothing consists of a convolution in time with a fixed wavelet $v_{\mathrm{pe}}(t)$ and a spatial convolution with a spatially varying $h_{\mathrm{pe}}\left(\mathbf{r}_{1}, \mathbf{r}_{5}, t\right)$.

\section{WAVE PROPAGATION EXPERIMENT}

This section compares the pressure field predicted by the model to a measured field to give an indication of the accuracy of the model for one scatterer embedded in a homogeneous medium. The measurement describes how a point is depicted by the transducer. This is done by moving a small point through the image plane. In practice, a point cannot be made and the tip of a needle is used to imitate a small point with a change in density and propagation velocity.

\section{A. Measurement conditions}

To make a precise comparison between theory and experiments, a concave, nonapodized transducer was used, for which analytic expressions are known for the spatial impulse response. The transducer used was a Brüel \& Kjaer type 8529 with a nominal frequency of $3.5 \mathrm{MHz}$. The focal radius is $150 \mathrm{~mm}$, and the radius of the element is $8.1 \mathrm{~mm}$.

The needle used had a radius of $0.3 \mathrm{~mm}$ and was made by removing the insulation from a copper wire. This should result in a smooth surface without spurious echoes from the needle surface so the scattered field only emanates from the tip of the needle. The needle then imitates a point in space. The needle was mounted on a fixture and was adjusted so that the needle was parallel to the acoustical axis of the transducer. The measurement was performed in a water bath of $850 \times 430 \times 500 \mathrm{~mm}$ (length, height, width). It contained distilled water that had been degassed for $24 \mathrm{~h}$.

A Brüel \& Kjaer 1846 ultrasound scanner was used to generate and receive the pulse to and from the transducer. During the measurements, the time gain amplifier in the scanner was set to linear amplification.

The high-frequency signal from the receiver amplifier was sampled by a LeCroy 9400 digital sampling oscilloscope, that has a resolution of 8 bits and an aperture jitter of $10 \mathrm{ps}$. The sampling frequency used was $100 \mathrm{MHz}$. The 3-dB bandwidth of the oscilloscope is $125 \mathrm{MHz}$. At full range, the signal-to-noise ratio is reported by the manufacturer to be $43.5 \mathrm{~dB}$ at $10 \mathrm{MHz}$. 
The scanner pulse emission was synchronized to the sampling frequency of the oscilloscope, making it possible to perform averaging on lines and to obtain coherency between lines at different positions. The synchronization also makes it possible to compare the measured and simulated lateral response. Each line shown is the average of ten measurements. This was done to reduce the measurement noise. The gain on the oscilloscope was set to the highest setting possible without clipping for each line, so the dynamic range could be fully utilized.

\section{B. Simulation model}

The simulation is based on the derived solution for the received pressure field, Eq. (45). During the simulation, we assume $f_{m}$ to be a Dirac impulse, corresponding to the needle used in the measurements, so (45) is reduced to

$$
p_{r}\left(\mathbf{r}_{5}, t\right)=v_{\mathrm{pe}}(t) * h_{\mathrm{pe}}\left(\mathbf{r}_{1}, \mathbf{r}_{5}, t\right)
$$

An analytic expression for $h$ is given by Arditi et al.$^{15}$ for a concave, nonapodized transducer.

To perform the calculations we must know $v_{\mathrm{pe}}$, as $h$ can be calculated from our knowledge of the physical dimensions of the transducer. So, $v_{\mathrm{pe}}$ can either be constructed from knowledge of the electromechanical properties of the transducer or it can be measured. We choose the second approach here, as this gives the most accurate determination of $v_{\text {pe }}$.

The response measured, when a pulse impinges on a planar reflector, is an amplitude-scaled replica of the original pulse, when the incident field is planar. The spatial impulse response at the focal point of a concave transducer is a Dirac impulse. Rewriting (49) we get

$$
\begin{aligned}
p_{r}\left(\mathbf{r}_{5}, t\right) & =\frac{1}{c_{0}^{2}} \frac{\partial^{2} v_{\mathrm{pe}}(t)}{\partial t^{2}} \star H_{\mathrm{pe}}\left(\mathbf{r}_{1}, \mathbf{r}_{5}, t\right) \\
& =\frac{1}{c_{0}^{2}} \frac{\partial^{2} v_{\mathrm{pe}}(t)}{\partial t^{2}} \star h\left(\mathbf{r}_{1}, \mathbf{r}_{5}, t\right) \star h\left(\mathbf{r}_{5}, \mathbf{r}_{1}, t\right)
\end{aligned}
$$

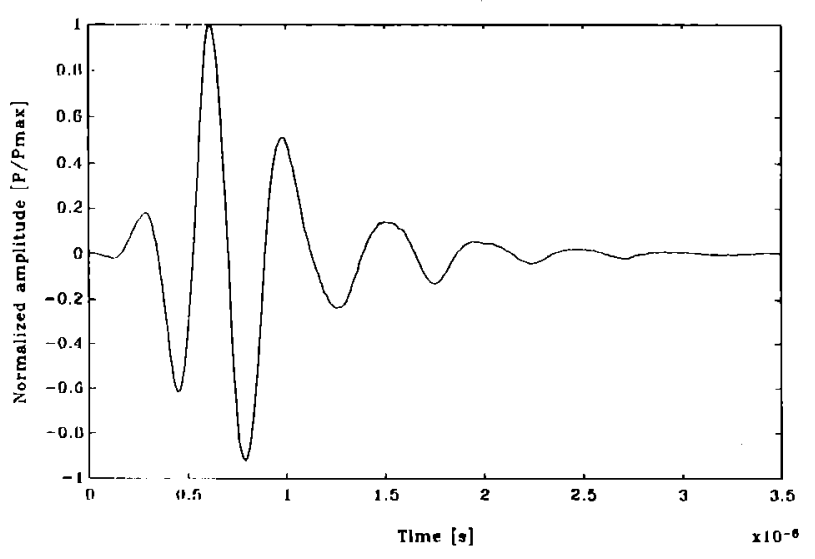

FIG. 4. Measured pulse-echo wavelet for the Brüel \& Kjaer 8529 transducer.

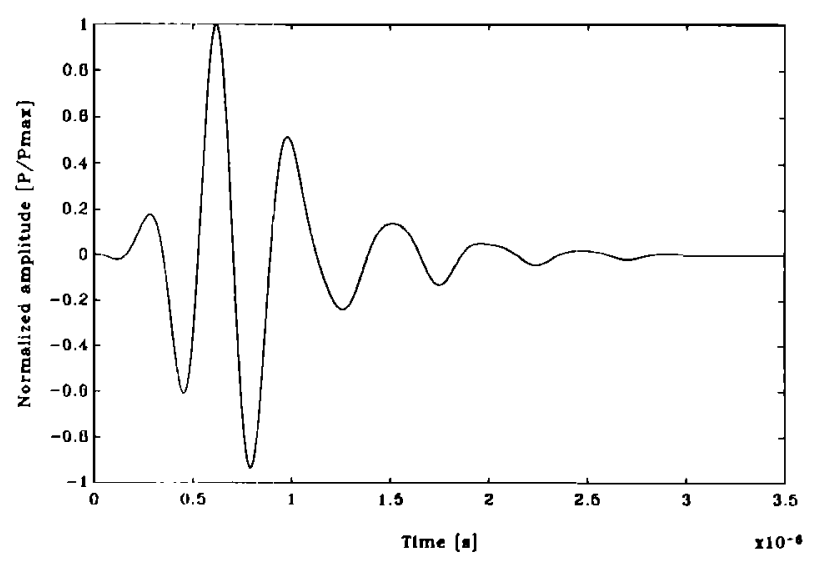

FIG. 5. Noise-filtered pulse-echo wavelet.

So, by placing a plane reflector at the focal point, we get the basic twice-differentiated pulse. This simple setup gives the pulse to be incorporated into the calculation of the field.

The pulse for the Brüel \& Kjaer 8529 transducer was measured by placing a perspex plate $150 \mathrm{~mm}$ from the transducer surface and then measuring the reflected pulse. The result is shown in Fig. 4. The measurement was performed by the LeCroy 9400 oscilloscope. The perspex plate was 10 $\mathrm{mm}$ thick and measured 150 by $500 \mathrm{~mm}$.

To remove noise, the response is filtered in the frequency domain by removing all components above $7 \mathrm{MHz}$, which results in the wavelet shown in Fig. 5. As seen, only the noise has been removed, thus this wavelet is used in the simulation.

When calculating $h$, it must be taken into account that $h$ has some abrupt changes, ${ }^{15,16}$ so to ensure a precise simulation, the time steps must be small. A value of 625 ps corresponding to a 1.6-GHz sampling frequency proved to be adequate.

\section{Comparison between measured and simulated responses}

The measured and simulated responses in Figs. 6 and 7 were obtained at a distance of $120 \mathrm{~mm}$ from the transducer surface. The measured pressure field was acquired by moving the needle in steps of $0.2 \mathrm{~mm}$ with an accuracy of 0.006 $\mathrm{mm}$, and measuring in a plane containing the acoustical axis of the transducer. Before the measuring, the transducer and the needle were aligned so that the needle was parallel to the acoustical axis. The data were sampled at a frequency of 100 MHz.

In Figs. 6 and 7, plots of the obtained responses are shown. The envelope of the RF-signals is shown as a mesh plot from two angles. The plots span 20-mm in the lateral direction and $4 \mu \mathrm{s}$ in the axial direction. A contour plot of the normalized logarithm of the envelope is also shown. The normalized if signal is shown at the acoustical axis, and at 3 $\mathrm{mm}$ from the acoustical axis. The normalized lateral re- 

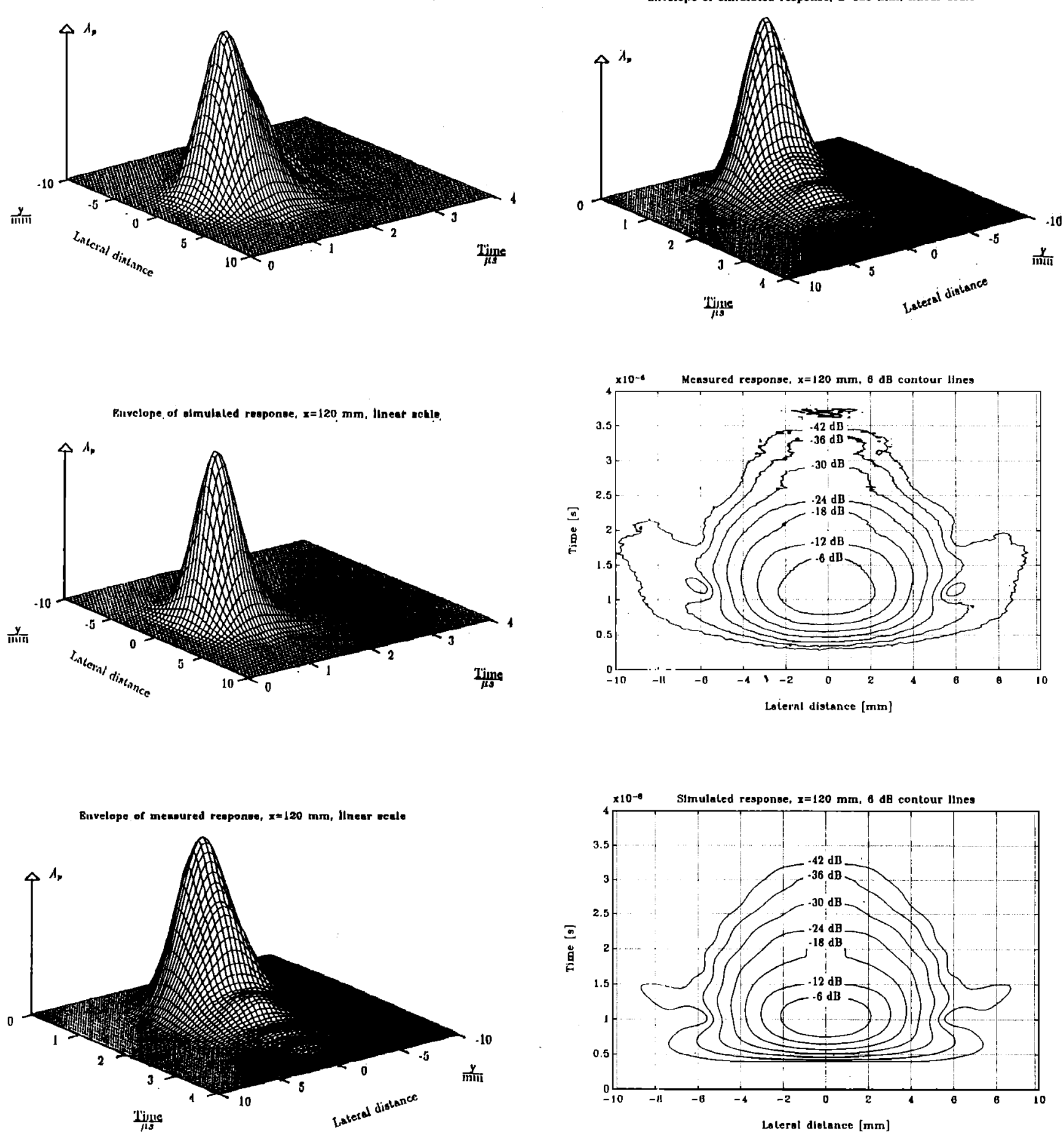

FIG. 6. Response at $x=120 \mathrm{~mm}$. Amplitude envelope plots.

sponse at the maximum positive peak of the response is also shown. The solid line is the measured response and the dashed line is the predicted response in Fig. 6. The left side of Fig. 7 shows the measured responses, the right side the simulated. A contour plot of the envelope measured and predict- ed at $x=60 \mathrm{~mm}$ is shown in Fig. 8 and at $x=200 \mathrm{~mm}$ in Fig. 9.

Comparing the measured and simulated responses, one sees that the model quite accurately predicts the measured responses. The main difference is the length of the responses, 

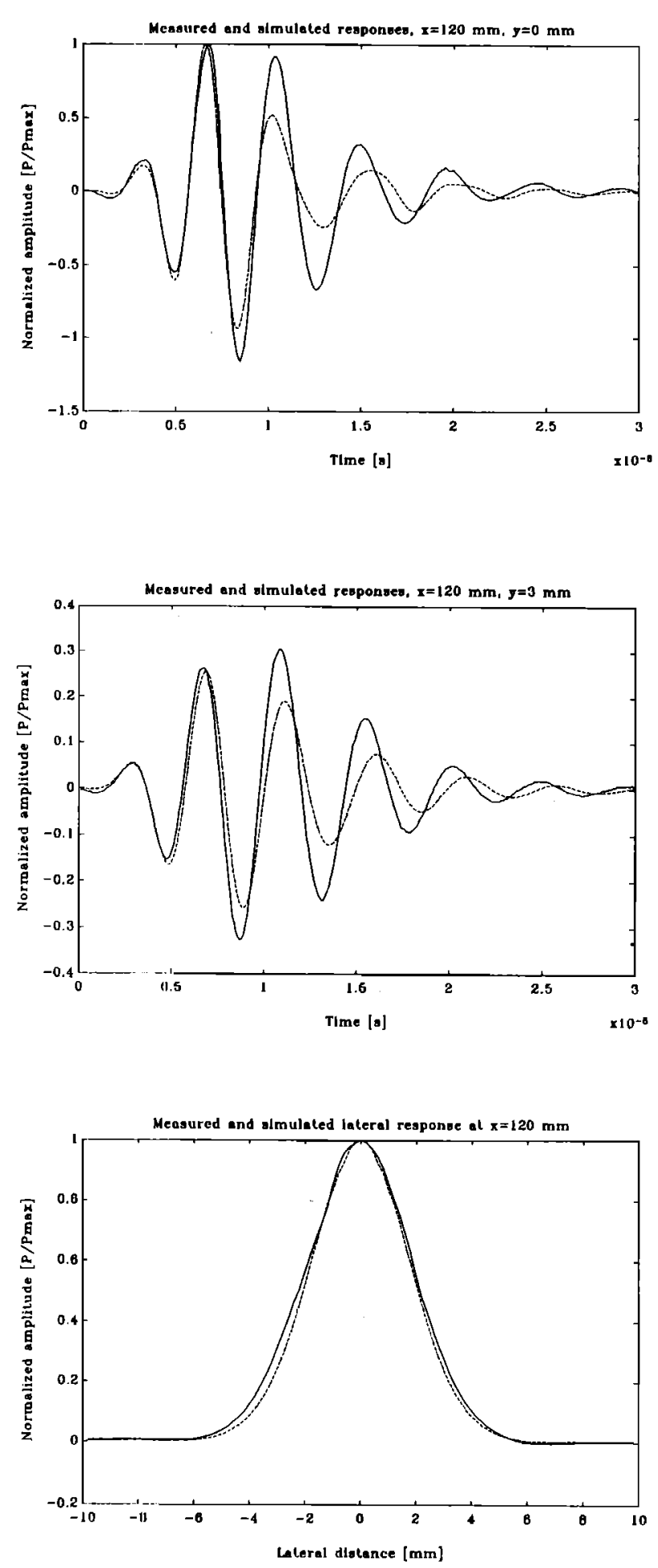

FIG. 7. Response at $x=120 \mathrm{~mm}$. Amplitude plots.

in which measured responses are consistently longer than predicted responses. This can possibly be ascribed to the shape of the needle, as there might have been some surface roughness or the tip had not been cut properly. It is also seen that the width of the responses differs from the predicted.
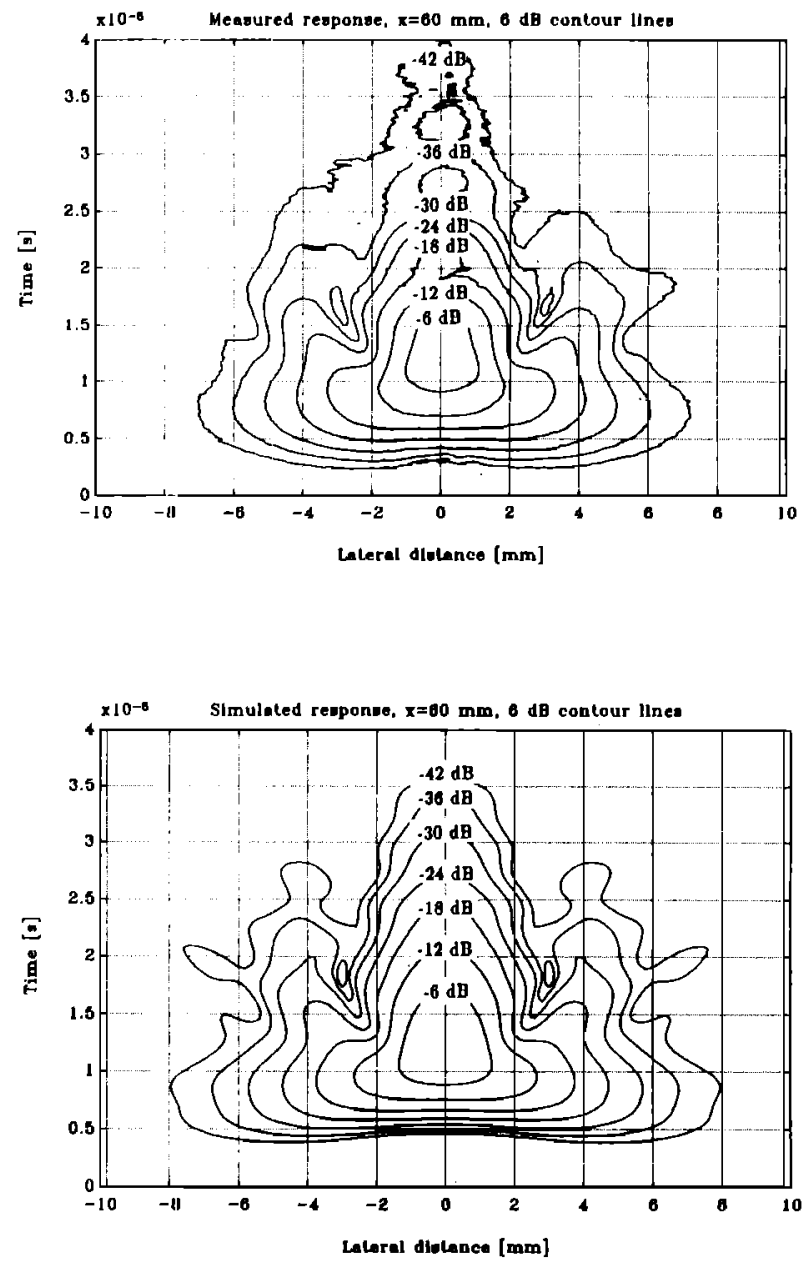

FIG. 8. Response at $x=60 \mathrm{~mm}$. Amplitude envelope plots.

This is due to the finite size of the needle, where in the simulation the scatterer was assumed to be a point.

These deviations are, however, small compared to the overall capability of the model to predict the actual response down to a level of -30 to $-40 \mathrm{~dB}$. So, in the case of no absorption and single scattering, the model derived is in good agreement with actual measurements. Further examples of measurements and simulations can be found in Jensen. ${ }^{16}$ They show the same pattern as the comparison given here.

\section{SUMMARY}

A wave equation for propagation of ultrasound in an inhomogeneous medium has been derived under the assumption of linear propagation and weak scattering. A general expression was derived for the received pressure field using the first-order Born approximation. The model can be used for a number of different transducer types and excitations. Analytic expressions for the spatial impulse response 

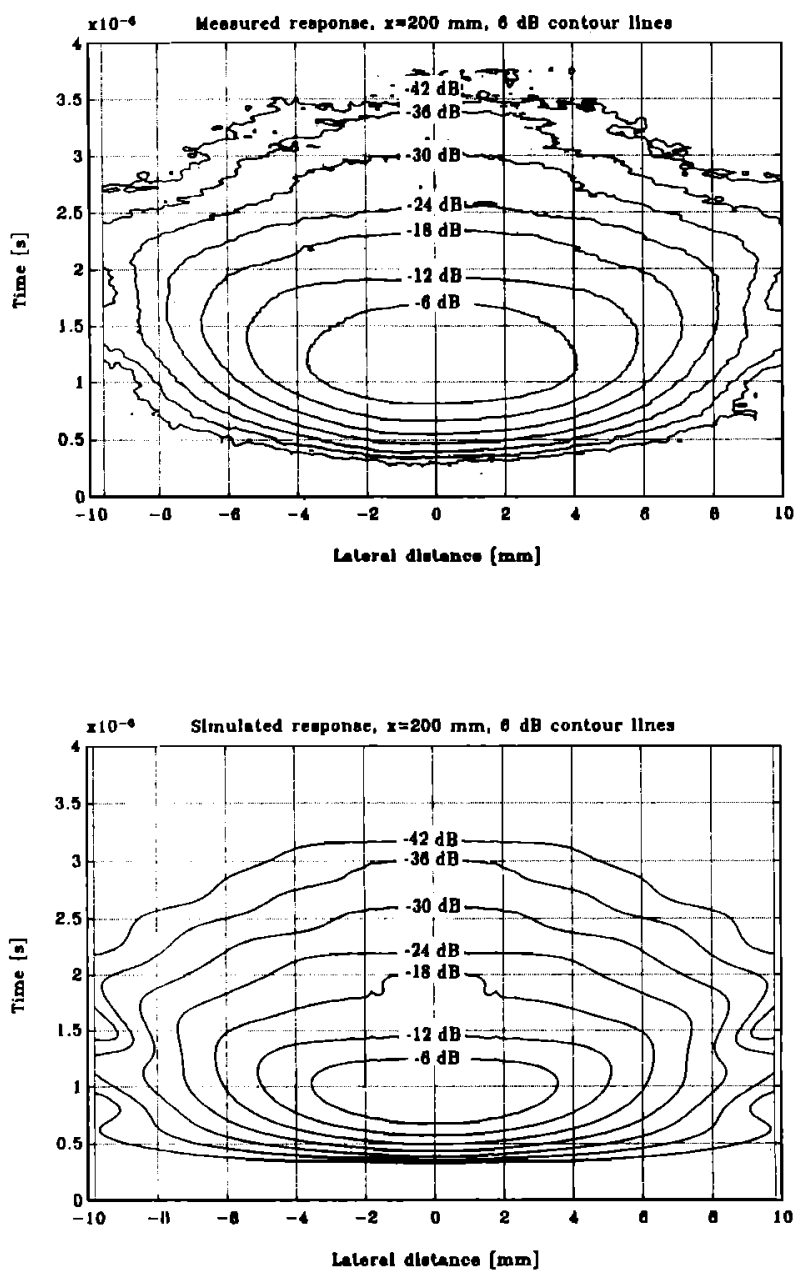

FIG. 9. Response at $x=200 \mathrm{~mm}$. Amplitude envelope plots.

for circular flat and concave, and rectangular transducers can readily be found in the literature.

The derived expression consists of three terms. The first, $f_{m}\left(\mathbf{r}_{1}\right)$ accounts for the density and propagation velocity perturbations in tissue. It is this parameter that should be displayed in medical ultrasound, but we observe a temporally and spatially smoothed version of the perturbations. The temporal smoothing is due to $v_{\text {pe }}$, which accounts for the excitation and the electromechanical transfer function of the transducer. The spatial smoothing is due to $h_{\mathrm{pe}}$, the modified pulse echo spatial impulse response resulting from the transducer geometry.
The model includes attenuation of the pulse due to propagation and scattering, but not the dispersive attenuation of the wave observed when propagating in tissue. This changes the pulse continuously as it propagates down through the tissue. Not including dispersive attenuation is, however, not a serious drawback of the theory, as this change of the pulse can be lumped into the already spatially varying $h_{\text {pe }}$. Or, if in the far field and assuming a homogeneous, dispersive attenuation, then an attenuation transfer function can be convolved onto $v_{\mathrm{pe}}$ to yield an attenuated pulse. Work is in progress in order to evaluate the accuracy of this approach.

\section{ACKNOWLEDGMENTS}

The author wishes to thank Dr. Sidney Leeman, King's College, London for useful discussions. The research was partly funded by the Danish Technical Research Council, Grant No. 16-4218. E, Brüel and Kjaer A/S, Novo's Foundation, H. C. Ørsteds Foundation, and Trane's Foundation.

${ }^{1} \mathrm{~J}$. C. Gore and S. Leeman, "Ultrasonic backscattering from human tissue: A realistic model," Phys. Med. Biol. 22, 317-326 (1977).

${ }^{2}$ M. Fatemi and A. C. Kak, "Ultrasonic B-scan imaging: Theory of image formation and a technique for restoration," Ultrason. Imag. 2, 1-47 (1980).

${ }^{3}$ L. A. Chernov, Wave Propagation in a Random Medium (McGraw-Hill, New York, 1960).

${ }^{4}$ P. M. Morse and K. U. Ingard, Theoretical Acoustics (McGraw-Hill, New York, 1968)

${ }^{5}$ S. Leeman, V. C. Roberts, P. E. Chandler, and L. A. Ferrari, "Inverse imaging with strong multiple scattering," in Mathematics and Computer Science in Medical Imaging, edited by M. A. Viergever and A. Todd-Pokropek (NATO ASI-series, Springer-Verlag, New York, 1988), pp. 279289.

${ }^{6} \mathrm{G}$. R. Harris, "Review of transient field theory for a baffled planar piston," J. Acoust. Soc. Am. 70, 10-20 (1981a).

${ }^{7}$ G. R. Harris, "Transient field of a baffled planar piston having an arbitrary vibration amplitude distribution," J. Acoust. Soc. Am. 70, 186-204 (1981b).

${ }^{8}$ G. E. Tupholme, "Generation of acoustic pulses by baffled plane pistons," Mathematika 16, 209-224 (1969).

${ }^{9}$ P. R. Stephanishen, "The time-dependent force and radiation impedance on a piston in a rigid infinite planar baffle," J. Acoust. Soc. Am. 49, 841849 (1971a).

${ }^{10} \mathrm{P}$. R. Stephanishen, "Transient radiation from pistons in a infinite planar baffle," J. Acoust. Soc. Am. 49, 1627-1638 (1971b)

${ }^{11}$ P. M. Morse and H. Feshbach, Methods of Theoretical Physics, Part I (McGraw-Hill, New York, 1953).

${ }^{12}$ A. Penttinen and M. Luukkala, "The impulse response and pressure nearfield of a curved ultrasonic radiator," J. Phys. D 9, 1547-1557 (1976).

${ }^{13} \mathrm{P}$. R. Stephanishen, "Pulsed transmit/receive response of ultrasonic piezoelectrical transducers," J. Acoust. Soc. Am. 69, 1815-1827 (1981).

${ }^{14}$ J. W. Hunt, M. Arditi, and F. S. Forster, "Ultrasound transducers for pulse-echo medical imaging," IEEE Trans. Biomed. Eng. BME-30, 453481 (1983).

${ }^{15}$ M. Arditi, F. S. Forster, and J. Hunt, "Transient fields of concave annular arrays," Ultrason. Imag. 3, 37-61 (1981).

${ }^{16} \mathrm{~J}$. A. Jensen, "Medical ultrasound imaging, an estimation based approach," Ph.D. dissertation, Electronic Institute, Technical University of Denmark (September 1988). 